\title{
Concurrent development of excitatory and inhibitory associations during backward conditioning
}

\author{
ROBERT W. TAIT and MICHAEL E. SALADIN \\ University of Manitoba, Winnipeg, Manitoba, Canada
}

\begin{abstract}
After backward pairings with a paraorbital shock, a tone CS was an effective punisher of licking in rabbits, yet yielded retarded forward acquisition of the rabbit's nictitating membrane response. The observation of both excitatory and inhibitory associative effects after a common training protocol challenges the assumption of a unidimensional associative continuum and supports approaches that assert a multidimensional structure to associations.
\end{abstract}

A continuing paradox for associative theories is the multiplicity of effects that result from following an aversive US with a CS. When the backward pairings are indirectly assessed with transfer-of-control paradigms, the CS usually suppresses an instrumental baseline (Burkhardt, 1980; Heth, 1976; Mowrer \& Aiken, 1954; Williams, Dyck, \& Tait, in press). This observation has led to the conclusion that backward conditioning produces excitatory associative effects. In contrast, when the effects of the backward pairings are assessed with a retardation-oflearning test, in which the CS and US are paired in a forward order and the development of CRs to the CS is monitored, the rate of CR acquisition is usually retarded (Plotkin \& Oakley, 1975; Siegel \& Domjan, 1971). The retarded acquisition has led to the conclusion that backward conditioning produces inhibitory associative effects (Hall, 1984).

In attempts to resolve the paradoxical effects of backward pairings, an association has usually been viewed as a unidimensional construct for which excitation and inhibition are mutually exclusive outcomes. As an association develops with backward pairings, a transient excitatory associative phase is thought to precede a terminal inhibitory association (Pavlov, 1932; Wagner \& Terry, 1975). Consequently, research strategies have emphasized functional relationships between associative strength and dynamic variables such as time, number of conditioning trials, and US-CS interval. Accordingly, one assessment procedure is selected, and variables that might affect associative strength are manipulated (Heth, 1976; Maier, Rapaport, \& Wheatley, 1976; Quesnel, Ten Have, \& Tait, 1980; Siegel \& Domjan, 1974). This strategy has produced reliable differences in the associative strength accrued to the backward CS, but not reliable evidence for

We thank C. D. Heth for his helpful comments and suggestions, D. A. Williams for developing the software for monitoring licking, and L. J. Quesnel for testing some of the animals. The research was supported by Grant AO312 from NSERC to R.W.T. Requests for reprints should be addressed to R. W. Tait, Psychology Department, University of Manitoba, Winnipeg, Manitoba, Canada R3T 2 N2. a change in the associative sign of the CS. That is, differences in excitatory or inhibitory associative strength have been observed, but not the expected mixture of excitatory and inhibitory effects.

In the present experiment, we adopted a different strategy in our approach to the problem. Instead of working with only one of the assessment procedures and varying the conditioning protocol, we maintained a fixed conditioning protocol and varied the assessment procedure. Rabbits were first trained to lick from a spout in an experimental chamber, then given backward conditioning of the nictitating membrane response. The associative effects of the backward CS were subsequently assessed first in a conditioned punishment paradigm and then in a retardation-of-learning test using the nictitating membrane response.

\section{METHOD}

\section{Subjects}

Thirty-five 2.5-kg male and female New Zealand white rabbits (Oryctolagus cuniculus) were individually housed and maintained on ad-lib feeding.

\section{Apparatus}

The $54 \times 43 \times 42 \mathrm{~cm}$ wooden lick chamber had a floor constructed from heavy-gauge wire mesh that was mounted above a floor pan. A 6-W houselight, an 8- $\Omega$ speaker, and the spout of a water bottle were positioned along one chamber wall. The water-bottle spout was modified so that the rabbit's tongue served to complete a circuit between the water and the lip of the spout. The signal from the completed circuit was amplified and read as a discrete computer input. All lick sessions terminated after the subjects had made 280 responses.

The apparatus for nictitating membrane conditioning has been described previously (Gormezano, 1966). Briefly, each rabbit was placed in a Plexiglas restraining box with its head firmly held by a pinnae clamp. A $10-\mathbf{k} \Omega$ microtorque rotary potentiometer was positioned on an adjustable head mount that was fitted firmly about the rabbit's snout. A piano-wire armature mechanically fixed to the shaft of the potentiometer was coupled to a $2.0-\mathrm{mm}$ loop sutured into the rabbit's right nictitating membrane. Movement of the nictitating membrane rotated the shaft of the potentiometer, and the resultant voltage changes were digitized and then analyzed by a soft- 
ware package (modified from Tait \& Gormezano, 1974) implemented on a Raytheon 703 computer.

For all conditioning sessions, the restraining boxes were placed in one of seven identical, ventilated, legal-size drawers of fireproof, sound-attenuating file cabinets. The front panel of each drawer contained a $6-W$ houselight, an $8-\Omega$ speaker, and two alligator leads for the delivery of shock. The alligator leads were clipped onto two Autoclip wound sutures implanted $10 \mathrm{~mm}$ caudal to and $10 \mathrm{~mm}$ above and below the horizontal plane of the rabbit's right eye. During all experimental sessions, 72-dB white noise was used in the experimental room to mask extraneous sounds. Extensions of $1.0 \mathrm{~mm}$ of the nictitating membrane were defined as responses.

\section{Procedure}

Two days after the rabbits arrived, a suture was implanted in the right nictitating membrane of each rabbit and a 200 -ml-of-waterper-day maintenance regimen started. Two days later, the experimental protocol was implemented. The experimental protocol consisted of five daily sessions of lick training, 1 day of classical conditioning, a lick recovery session, and a conditioned punishment test session in the lick apparatus, followed by a retardationof-learning test in the classical conditioning chambers.

Parameter selection for the experiment was complicated by the absence of known effective parameters of conditioned punishment for the rabbit. Therefore, the parameters that were selected were a compromise between backward conditioning parameters used in conditioned punishment of rats and those used in nictitating membrane response conditioning in the rabbit. In conditioned punishment, effective backward CSs have had durations of $1 \mathrm{sec}$ or longer (e.g., Heth, 1976; Heth \& Rescorla, 1973), whereas, in nictitating membrane response conditioning, backward CS durations have been less than 1 sec (e.g., Plotkin \& Oakley, 1975; Siegel \& Domjan, 1971). In an attempt to map into both empirical literatures, we chose a CS duration of $1 \mathrm{sec}$. A more critical problem was the number of pairings needed to produce reliable effects. In conditioned punishment, excitatory conditioning produced by both forward and backward pairings appears to yield a nonmonotonic acquisition function (Heth, 1976; Sherman \& Maier, 1978). With a large number of pairings (80-100), there is a lower likelihood of identifying excitatory effects and, therefore, differential excitatory effects. On the other hand, inhibitory backward conditioning of the nictitating membrane response reportedly requires a large number of pairings (125 in the case of Plotkin \& Oakley, 1975; 495 in the case of Siegel \& Domjan, 1971). However, when the backward acquisition function was examined (Quesnel, Ten Have, \& Tait, 1980), reliable inhibitory backward conditioning emerged after 65 backward pairings when a retardation-of-learning test with a CS-US interval of $500 \mathrm{msec}$ was subsequently employed to assess the pairings. Therefore, to stay within the effective range of parameters for the two conditioning paradigms, we used the backward conditioning parameters (with the exception of CS duration) and the retardationof-learning parameters employed by Quesnel et al. (1980).

During the classical conditioning session, the subjects were assigned randomly to one of five treatments $(n=7)$. The forward (FWD) group received 65 forward pairings of a $1,000-\mathrm{msec}, 1000$ $\mathrm{Hz}, 80-\mathrm{dB}$ tone and a $100-\mathrm{msec}, 2.5-\mathrm{mA}, 60-\mathrm{Hz}$ ac paraorbital shock at a CS-US interval of $500 \mathrm{msec}$. The backward (BKD) group received 65 shock-tone pairings with shock onset preceding tone onset by $500 \mathrm{msec}$. The CS-alone (CS) group received 65 tone presentations, and the US-alone (US) group received 65 shock presentations. The final group (Group $N$ ) was restrained and placed in the file drawers for a time equivalent to the session length of the other groups but received no stimuli. All intertrial intervals averaged 120 sec.

The conditioned punishment test was identical for all subjects. The punishment period was initiated by the 140th lick. Each of the next 20 licks was followed by the presentation of the tone for $1,000 \mathrm{msec}$. If a subject made a licking response during the tone, an additional 1,000 msec was added to the duration of the tone so that at the end of the punishment period, all subjects had received the same duration of exposure to the tone. The time to make the 20 licks was measured as the dependent variable. The retardation test consisted of four daily sessions in which all rabbits received 50 pairings of a $550-\mathrm{msec}, 1,000-\mathrm{Hz}, 80-\mathrm{dB}$ tone and a $50-\mathrm{msec}$, $2.5-\mathrm{mA}, 60-\mathrm{Hz}$ paraorbital shock, with the offsets of the tone and shock coterminous. The mean intertrial interval was $60 \mathrm{sec}$. The percent CRs measured during the 500-msec CS-US interval and summed over the 4-day test was the dependent variable. The order of the two tests was selected on the basis of pilot work, which showed that the presentation of $20 \mathrm{CSs}$ in a different environment had no detectable effect on a subsequent retardation-of-learning test.

\section{RESULTS}

During the backward conditioning phase, the overall percentages of CRs were $5.2,2.4,2.9,1.8$, and 2.0 for Groups FWD, BKD, N, CS, and US, respectively. The low level of responding precluded analysis. An examination of the last five trials of the session showed that Group FWD demonstrated some acquisition (20\%), but that Group BKD (2.9\%) remained at the levels of the control groups $(2.9 \%, 3.0 \%$, and $3.4 \%$ for Groups $N, C S$, and US, respectively).

The left-hand frame of Figure 1 displays the conditioned punishment test results for Groups FWD, BKD, N, CS, and US and shows that Groups FWD and BKD took substantially longer to complete 20 licks than did the control groups. An ANOVA yielded a significant groups effect $[F(4,30)=3.28, p<.05]$, which, as shown by post hoc orthogonal contrast tests, resulted from the longer times of Groups FWD and BKD relative to Groups N, CS, and US $[F(1,30)=11.15, p<.01]$. Groups FWD and BKD did not differ, and no differences existed between the control groups.

The longer times for Groups FWD and BKD relative to control groups indicate that the CS in the two groups was an effective punisher. Accordingly, the backward and forward conditioning treatments yielded excitatory associative effects.

The right-hand frame of Figure 1 displays the mean percent NM CRs on the retardation test for Groups FWD, BKD, N, CS, and US. The figure indicates that Group FWD had a higher, and Group BKD a lower, mean percent NM CR than the other groups. Again, the ANOVA revealed a significant group effect $[F(4,30)=$ $4.72, p<.01]$. Post hoc orthogonal contrasts showed that the percentage of NM CRs was higher for Group FWD than for Groups N, CS, and US, $[F(1,30)=8.30, p<$ $.01]$, and that the percentage of NM CRs was lower for Group BKD than for Groups N, CS, and US $[F(1,30)=$ $4.52, p<.05]$. No differences were detected between the control groups.

\section{DISCUSSION}

The higher percentage of NM CRs for Group FWD documents a positive transfer from the initial forward conditioning treatment to the retardation test. Thus, the ini- 

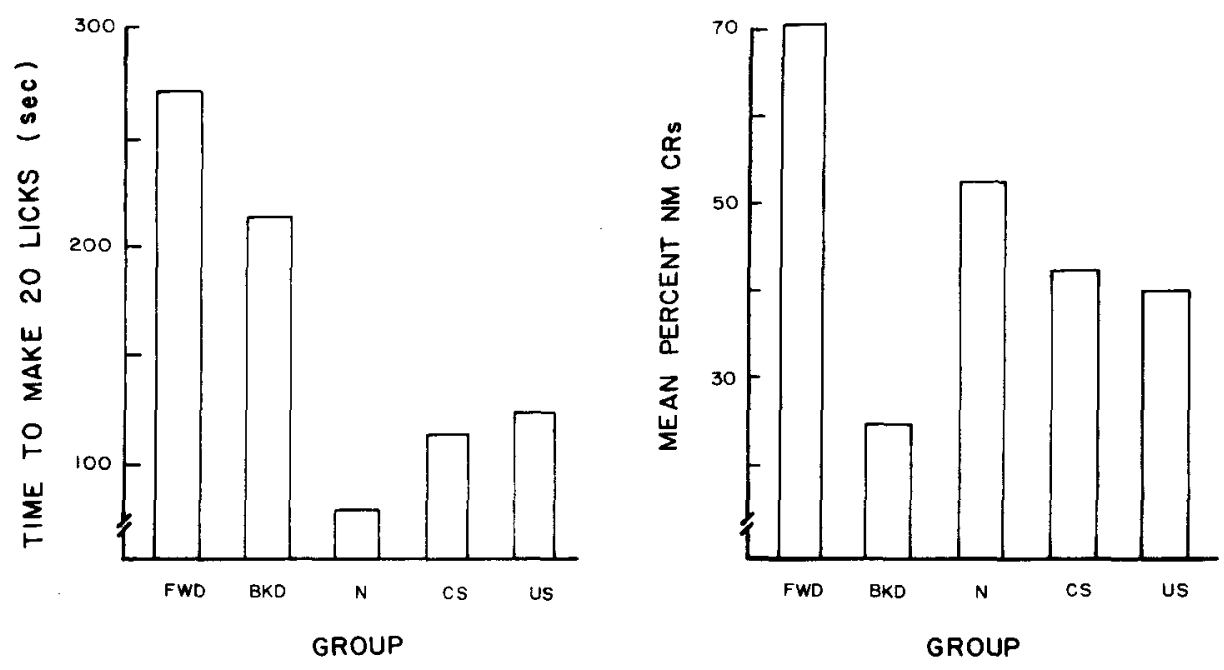

Figure 1. The left-hand frame depicts the mean time to make 20 licks on the conditioned punishment test; the right-hand frame shows the mean percentage of NM CRs on the retardation test for Groups FWD, BKD, N, CS, and US.

tial forward treatment produced excitatory associative effects, and the outcome of the retardation test is consistent with the interpretation of the results of the conditioned punishment test. In contrast, the lower percentage of NM CRs for the backward group indicates that retarded acquisition occurred, and therefore a negative transfer from the initial backward conditioning treatment was obtained. The retarded acquisition, which replicates previous observations with the NM response (Quesnel et al., 1980; Plotkin \& Oakley, 1975; Siegel \& Domjan, 1971), leads to the conclusion that the backward treatment produced associative inhibition. This conclusion is directly opposite to the interpretation of the conditioned punishment test results.

The paradoxical effects of backward conditioning in the present study replicate observations reported in the aversive conditioning literature. Most transfer-of-control experiments, and all conditioned punishment tests, report excitatory outcomes for backward conditioning (Burkhardt, 1980; Heth, 1976; Mowrer \& Aiken, 1954; Williams et al., in press). In contrast, retardation-of-learning tests employed with classical conditioning paradigms yield inhibitory effects (Hall, 1984; Plotkin \& Oakley, 1975; Siegel \& Domjan, 1971). Since the species studied, conditioning procedures, and conditioning parameters varied greatly between the two sets of experimental results, direct comparisons of the contradictory findings were not possible. However, in the present experiment, the conditioning parameters were held constant and the assessment procedures were varied. Thus, the present observation of both excitatory and inhibitory effects with backward pairings bridges the two sets of empirical results by confirming the presence of both effects after a common treatment.

The dissociation of two different outcomes under one set of conditioning parameters is not a novel observation.
Differences in rates of acquisition between classically conditioned responses (e.g., heart rate) and the suppression of instrumental behavior by the same CS have been noted previously (e.g., de Toledo \& Black, 1966; Parrish, 1967). Furthermore, differential sensitivity to common sets of classical conditioning parameters have been noted for different response systems (see Schneiderman, 1972, for a systematic examination of parameters which produce divergencies between nictitating membrane and heart rate conditioned responses). However, previous observations have focused on identifying differences either in rates of acquisition or in parameters that lead to the conditioning of one response system and not another. The present study extends the existing literature by revealing apparent opposite associative outcomes for different response systems after a common conditioning history.

The observation of both excitatory and inhibitory associative effects produced by the same CS as the consequence of a single training regime is contrary to the expectations of unidimensional models of associative processes (Moore \& Stickney, 1985; Pearce \& Hall, 1980; Rescorla \& Wagner, 1972). Unidimensional models assert that the associative strength of a CS can be viewed as a continuum ranging from positive to negative values, and that a training protocol involving that CS may cause an increase or decrease in its associative strength, which can be mapped by changes in observed performance. According to these theories, an invariance in the ordering of behavioral indices of the association should be observed after a common training protocol. Thus, if the associative strength is positive, excitatory associative effects should be uniformly observed. Conversely, if the associative strength is negative, inhibitory effects should appear uniformly. Since the theories do not allow for the simultaneous occurrence of both a positive and a negative as- 
sociative strength to a CS, a unidimensional theoretical approach cannot account for the concurrent excitatory and inhibitory effects identified in the present study.

Rather, our results suggest that associations formed during classical conditioning are multidimensional constructs. Similar views of the consequences of classical conditioning have been assumed by mediational theories of instrumental conditioning that assert that a CS may acquire both a cuing and a motivational mediational role (e.g., Konorski, 1967; Overmier \& Lawry, 1979; Spence, 1956). Empirical support for the dual consequences of classical conditioning is provided by the results of classical-classical transfer of training experiments in which a CS is first paired with a US from one class of hedonic US and later paired with a hedonically opposite US. Acquired motivational effects are implicated by the retarded acquisition observed with the hedonically opposing US (e.g., Scavio, 1974). Acquired cuing effects are implicated by: (1) the savings observed during the reacquisition of the original CR using the initial US (Bromage \& Scavio, 1978); (2) the ability of a US to function as a CS when directly paired with a hedonically opposite US (see Gormezano \& Tait, 1976); and (3) the simultaneous occurrence of hedonically opposite responses to a CS following an acquisition sequence in which the CS is paired with a US, which is subsequently paired with a hedonically opposite US (Tait, Quesnel, \& Ten Have, 1986). Furthermore, it has been asserted (e.g., Soltysik, 1971) that the two associative consequences may be differentially sensitive to the effects of classical conditioning variables. The results of the present experiment are consistent with this assertion, because, with the backward conditioning parameters employed, excitatory conditioning of the motivational consequence of conditioning, but inhibitory conditioning of the cuing consequence occurred.

Viewing an association as a multidimensional construct does not readily lead to the expectation that associations have the potential for coexisting excitatory and inhibitory components. Moreover, the present results do not indicate how the components are to be conceptualized. Two approaches are plausible. The first would assert a common set of associative processes and would predict different behavioral outcomes based on differences in response dynamics. The second would assert that the on-trial temporal dynamics of stimulus processing yield multiple associative components. The former approach is taken by opponent process theory (Solomon, 1980; Solomon \& Corbit, 1974), the latter by Wagner's (1981; Mazur \& Wagner, 1984) SOP model.

In opponent process theory, it is assumed that a US produces a reaction ( $a$ process) that initiates a compensatory counterreaction ( $b$ process) in affected response systems. It is further assumed that different response systems will have different temporal courses for the $a$ and $b$ processes, and therefore to the subtractive interaction between the two processes. If it is assumed that conditioning is acquired through contiguity (e.g., Schull, 1979), then it is possible that the following two conditions devel- oped during the experiment. For the nictitating membrane response, the backward CS occurred when the $b$ processes were stronger than the $a$ processes. In contrast, for the motivational states that purportedly regulate conditioned punishment behavior (Rescorla \& Solomon, 1967), the $b$ processes were weaker than the $a$ processes at the time of CS presentation. If both conditions occurred, then inhibitory effects would be observed with the nictitating membrane response and excitatory effects would be observed with conditioned punishment.

In the SOP model, it is assumed that memorial elements of a US (the elements may reflect stimulus and motivational characteristics of the US) are activated at US onset and then decay through two states to an inactive state. If a CS occurs during the decay of elements in the US memorial node, then associations between the CS and US notes would be established. An excitatory association results if most of the elements in the US node are in the temporally first active states, whereas an inhibitory association develops if most of the elements have decayed to the temporally second active state. If there are elements in both active states, then both excitatory and inhibitory components to the association would be formed. Although the model allows for both excitatory and inhibitory components to the association, it provides little rationale for why one response system would reflect the excitatory component while a different response system reflected the inhibitory component.

\section{REFERENCES}

Bromage, B. K. , SCAvio, M. J. (1978). Effects of aversive CS+ and CS- under deprivation upon successive classical appetitive and aversive conditioning. Animal Learning \& Behavior, 6, 57-65.

BurkHARDT, P. E. (1980). One trial backward fear conditioned as a function of US intensity. Bulletin of the Psychonomic Society, 15, 9-11.

de Toledo, L., \& Black, A. H. (1966). Heart rate: Changes during conditioned suppression in rats. Science, 152, 1404-1406.

Gormezano, I. (1966). Classical conditioning. In J. B. Sidowski (Ed.), Experimental methods and instrumentation in psychology. New York: McGraw-Hill.

Gormezano, I., \& TAIT, R. W. (1976). The Pavlovian analysis of instrumental conditioning. Pavlovian Joumal of Biological Science, 11 $37-55$.

HALL, J. F. (1984). Backward conditioning in Pavlovian type studies: Reevaluation and present status. Pavlovian Journal of Biological Science, 19, 163-169.

HETH, C. D. (1976). Simultaneous and backward fear conditioning as a function of the number of CS-UCS pairings. Journal of Experimental Psychology: Animal Behavior Processes, 2, 117-129.

Heth, C. D., \& Rescorla, R. A. (1973). Simultaneous and backward fear conditioning in the rat. Journal of Comparative \& Physiological Psychology, 82, 434-443.

KONORSKI, J. (1967). Integrative activity of the brain: An interdisciplinary approach. Chicago: University of Chicago Press.

MaIer, S. F., RAPAPORT, P., \& Wheatley, K. L. (1976). Conditioned inhibition and the UCS-CS interval. Animal Learning \& Behavior, 4, $217-220$

Mazur, J. E., \& Wagner, A. R. (1984). An episodic model of associative learning. In M. Commons, R. Hermstein, \& A. R. Wagner (Eds.), Quantitative analyses of behavior acquisition (Vol. 3). Cambridge, MA: Hallinger.

MoORE, J. W., \& STICKNEy, K. J. (1985). Antiassociations: Conditioned inhibition in attentional-associative networks. In R. R. Miller \& N. E. 
Spear (Eds.), Information processing in animals: Conditioned inhibition. Hillsdale, $\mathrm{NJ}$ : Erlbaum.

Mowrer, D. H., \& AIKEN, E. G. (1954). Contiguity vs. drive reduction in conditioned fear: Temporal variations in conditioned and unconditioned stimulus. American Journal of Psychology, 67, 26-38.

OVermier, J. B., \& LAWRY, J. A. (1979). Pavlovian conditioning and the mediation of behavior. In G. H. Bower (Ed.), The psychology of learning and motivation (Vol. 13). New York: Academic Press.

PARRISH J. (1967). Classical discrimination conditioning of heart rate and bar-press suppression in the rat. Psychonomic Science, 9, 267-268.

Pavlov, I, P. (1932). The reply of a physiologist to psychologists. Psychological Review, 39, 91-127.

Pearce, J. M., \& Hall, G. (1980). A model of Pavlovian learning: Variations in the effectiveness of conditioned but not unconditioned stimuli. Psychological Review, 87, 532-552.

PlotKin, H. C, OAKLEY, D. A (1975). Backward conditioning in the rabbit (Oryctolagus cuniculus). Journal of Comparative \& Physiological Psychology, 88, 586-590.

Quesnel, L. J., Ten Have, W. N., \& TAIT, R. W. (1980). Backward acquisition curves for the classical aversive and appetitive responses in the rabbit (Oryctolagus cuniculus). Paper presented at the Canadian Psychological Association meetings, Calgary.

Rescorla, R. A., \& Solomon, R. L. (1967). Two-process learning theory: Relationship between Pavlovian conditioning and instrumental learning. Psychological Review, 74, 151-182.

Rescorla, R. A., \& WAGNer, A. R. (1972). A theory of Pavlovian conditioning: Variations in the effectiveness of reinforcement and nonreinforcement. In A. H. Black \& W.F. Prokasy (Eds.), Classical conditioning II: Current research and theory. New York: AppletonCentury-Crofts.

SCAvio, M. J. (1974). Classical-classical transfer: Effects of prior aversive conditioning upon appetitive conditioning in rabbits. Journal of Comparative \& Physiological Psychology, 86, 107-115.

SCHNEIDERMAN, N. (1972). Response system divergencies in aversive classical conditioning. In A. H. Black \& W. F. Prokasy (Eds.), Classical conditioning II: Current theory and research. New York: Appleton-Century-Crofts.

SchULL, J. (1979). A conditioned opponent theory of conditioning and habituation. In G. H. Bower (Ed.), The psychology of learning and motivation (Vol. 13). New York: Academic Press.

Sherman, J. E., \& Maier, S. F. (1978). The decrement in conditioned fear with increased trials of simultaneous conditioning is not specific to the simultaneous procedure. Learning \& Motivation, 9, 31-53.

Siegel, S., \& DomJan, M. (1971). Backward conditioning as an inhibitory procedure. Learning \& Motivation, 2, 1-11.

Siegel, S., \& Domjan, M. (1974). The inhibitory effect of backward conditioning as a function of the number of backward pairings. Bulletin of the Psychonomic Society, 4, 122-124.

SoLomon, R. L. (1980). Recent experiments testing an opponent-process theory of acquired motivation. Acta Neurobiologiae Experimentalis, 40, 271-289.

Solomon, R. L., \& CoRBi, J. D. (1974). An opponent-process theory of motivation: Temporal dynamics of affect. Psychological Review, 81, 119-145.

SoLTYSIK, S. (1971). On the homogeneous and heterogeneous transformation of conditioned reflexes: A comment on the Overmier and Payne case of positive transfer. Acta Neurobiologiae Experimentalis, 31, 401-407.

SPENCE, K. W. (1956). Behavior theory and conditioning. New Haven: Yale University Press.

TAIT, R. W., GORMEZANO, I. (1974). A microcomputer program for stimulus control and analog data for discrete trial paradigms in biological preparations: Classical conditioning. Behavior Research Methods \& Instrumentation, 6, 295-300.

TAIT, R. W., Quesnel, L. J., \& TEN HAVE, W. N. (1986). Classicalclassical transfer: Excitatory associations between "competing" motivational stimuli during classical conditioning of the rabbit. Animal Learning \& Behavior, 14, 138-143.

WAGNER, A. R. (1981). SOP: A model of automatic memory processing in animal behavior. In N. E. Spear \& R. R. Miller (Eds.), Information processing in animal memory mechanisms. Hillsdale, $\mathrm{NJ}$ : Erlbaum.

WAGNER, A. R., \& TERRY, W. (1975). Backward conditioning to a CS following an expected vs a surprising UCS. Animal Learning \& Behavior, 3, 370-374.

Williams, D. A., DyCK, D. G., \& TAIT, R. W. (in press). Excitatory backward conditioning in conditioned punishment and conditioned suppression. American Journal of Psychology.

(Manuscript received September 26, 1985; revision accepted for publication March 3, 1986.) 